\title{
A review of endoscopic surgical applications in oral and maxillofacial surgery
}

\author{
Christopher A. Fanelli ${ }^{1}$, Luke C. Vera ${ }^{2}$, David Y. Ahn ${ }^{2}$, Joseph P. McCain ${ }^{1}$ \\ ${ }^{1}$ Department of Oral and Maxillofacial Surgery, Harvard School of Dental Medicine, Harvard Medical School, Massachusetts General Hospital, \\ Boston, MA, USA; ${ }^{2}$ Department of Oral and Maxillofacial Surgery, David Grant USAF Medical Center, Fairfield, CA, USA \\ Contributions: (I) Conception and design: CA Fanelli, JP McCain; (II) Administrative support: All authors; (III) Provision of study materials or \\ patients: JP McCain; (IV) Collection and assembly of data: CA Fanelli, DY Ahn; (V) Data analysis and interpretation: All authors; (VI) Manuscript \\ writing: All authors; (VII) Final approval of manuscript: All authors. \\ Correspondence to: Christopher A. Fanelli. Department of Oral and Maxillofacial Surgery, Harvard School of Dental Medicine, Harvard Medical \\ School, Massachusetts General Hospital, 55 Fruit Street, Warren 1201, Boston, MA 02114, USA. Email: CAFANELLI@mgh.harvard.edu.
}

\begin{abstract}
This article provides an updated review of oral and maxillofacial endoscopic surgical applications in craniomaxillofacial trauma management, orthognathic surgery, sialoendoscopy, open temporomandibular joint (TMJ) arthrotomy procedures, and minimally invasive arthroscopic TMJ surgery. The integration of surgical endoscopy into oral and maxillofacial procedures has yielded a seminal impact on achieving comparable or improved patient outcomes relative to open standard operations in numerous areas of maxillofacial surgery. Maxillofacial endoscopy provides assisted visualization and surgical enhancement to many classic open surgical operations. Alternatively, endoscopic maxillofacial surgical applications can be implemented as a minimally invasive ("closed") surgical option that serves to decrease patient morbidity, shorten hospital admissions, and optimize expedited recovery and healing post-operatively. As a narrative review, this article integrates and details the key findings of the available literature on maxillofacial endoscopic surgery to provide a concise review on the subject from the background and history to a summary of each of the surgical techniques and the available outcome data on each procedure. As endoscopic maxillofacial surgery continues to gain popularity and increased global implementation due to its continued demonstrated efficacy and positive results, continual review of current techniques, applications, and outcomes are integral to the ongoing innovation and advancement of this dynamic subspecialty of maxillofacial surgery.
\end{abstract}

Keywords: Endoscopic; minimally invasive surgery; maxillofacial surgery; trauma; temporomandibular joint (TMJ); orthognathic

Received: 02 February 2021; Accepted: 26 November 2021; Published online: 14 December 2021.

doi: $10.21037 /$ fomm-21-12

View this article at: https://dx.doi.org/10.21037/fomm-21-12

\section{Introduction \& history}

Endoscopy can be broadly defined as the ability to visualize internal structures within the body using an illuminated, navigable instrument called an endoscope which can be introduced via a natural orifice or surgical incision (1). In 1806, Phillipp Bozzini produced the first internally lit endoscope which he coined "Lichtleiter" by implementing a candle and a system of angled mirrors for observation (2). The term endoscope subsequently came to prominence in
1853 when Antonin Jean Desormeaux applied this novel technology to perform endoscopic urological surgery. He coined the term "endoscope" (3). Employment of this minimally invasive, novel technology continued with application to other anatomical structures of the body. The introduction of the endoscope into a joint cavity known as "arthroscopy" was first reported in the literature by Dr. Eugen Bircher in 1921. Bircher introduced a laparoscope within the knee, and achieved an intra-articular optical cavity by distending the joint utilizing nitrogen gas (4). 
The initial application of endoscopic surgery in oral and maxillofacial surgery was conducted by Japanese surgeon Masatoshi Onishi, who was the first to conduct temporomandibular joint (TMJ) arthroscopy. Subsequent pioneering oral and maxillofacial surgeons that further established and expounded upon diagnostic and operative TMJ arthroscopy include: Murakami, McCain, Sanders, and Holmlund (5). Maxillofacial endoscopic surgical applications were further elaborated upon in conducting sialoendoscopy in the treatment and management of salivary gland obstruction. Early innovators include Marchal, Nahlieli, Katz, and Konisgsberger who established endoscopic surgical applications and techniques to salivary gland orifices trans-orally (6). Most recently, maxillofacial endoscopy has been utilized to conduct minimally invasive surgical approaches to the ramus condyle unit (RCU), reduction and internal fixation of subcondylar and ramal fractured osseous segments of the RCU and associated trauma applications by Mueller, Schmelzeisen, Kellman, Troulis, Kaban, and Lee have been established $(7,8)$.

\section{General endoscopic principals and advantages}

\section{Surgical education and documentation}

As an instrument for teaching, endoscopically assisted surgery allows trainee surgeons to easily and directly visualize the operation on the monitor. The teaching surgeons are enabled to clearly describe the procedure in real time, while also are able to easily capture images that can be later used for subsequent education and research $(9,10)$. A common effect of conducting endoscopic surgery is increased attention of all individuals in the operating room, as all members can be engaged with the happenings and nuances of the operation being conducted with excellent visualization on the monitors. Use of the endoscope also allows for efficient documentation of critical aspects of all operations via photo and video image capture. This prevents the need for over-gloves or photobags to be placed over the surgeon's hands when handling a non-sterile camera. The risk of surgical site infection due to contamination from using a non-sterile camera is eliminated with the use of a sterile endoscope used during the procedure as well. Photo and video documentation can be useful from a medicolegal standpoint.

\section{Patient recovery and surgical safety outcomes}

Patient outcome studies evaluating endoscopic surgical procedures have demonstrated reduced patient morbidity, shorter hospital admission times, and expedited healing and recovery with return to normal function when compared with standard open surgical techniques (11). The physiological basis of this concept has been demonstrated in animal models comparing maxillofacial endoscopic surgical approaches which yielded significantly less postoperative edema when compared with traditional open surgical approaches (12). It stands to reason that the use of endoscopy allows for the use of small incisions which reduces tissue violation and manipulation, and may allow for faster recovery and reduced postoperative discomfort when compared with some traditional open surgical techniques.

A greater margin of safety when conducting maxillofacial procedures with the use of an endoscope has been suggested. This is due to improved visualization of structures and enhanced diagnosis that may assist in the avoidance and prevention of technical error $(13,14)$.

\section{Endoscopic armamentarium and general concepts}

The components of the endoscopic equipment setup include the endoscopy tower which is composed of the camera, a light source, and a video processor. Other necessary components include a monitor, endoscopic coupler, and the endoscope itself.

The optical characteristics of the endoscope include the field of view: the angle created from the tip of the rigid endoscope to the extreme edge of the field. The direction of view is the angle projected between the long axis of the rigid endoscope and the line through the center of the image being visualized. The angulation of the scope typically relates to the latter. Endoscopes most commonly utilized in head and neck endoscopy are $0^{\circ}, 10^{\circ}, 30^{\circ}, 45^{\circ}$, and $70^{\circ}$. Focal length is defined as the distance that is in focus, between the object and the distal tip of the scope. The optical cavity is the anatomical space where the endoscope is inserted to visualize and operate. Optical cavities exist in the body as natural cavities (e.g., the maxillary sinus), surgically created (e.g., a subperiosteal space), or a combination of these two principles (e.g., insufflated of a joint space as in arthroscopy).

Three main categories of endoscopes exist which include: flexible, rigid, and semirigid. Flexible endoscopes are utilized for procedures such as laryngoscopy and gastrointestinal endoscopy.

Rigid endoscopes are the most commonly used scope in maxillofacial endoscopic surgery, and are used in conducting 
arthroscopy, sinuscopy, and rhinoscopy. Semirigid endoscopes are commonly used in sialoendoscopy (15).

\section{Review of applications in traumatic maxillofacial injuries}

\section{Orbital floor reconstruction}

The endoscopic approach to orbital floor reconstruction can be applied as a trans-antral only approach, or it may be used in combination with the open approach. The benefits of the endoscopic approach are in line with its benefits in other applications, notably, improved visualization along with a simultaneous reduction in the surgical insult to the tissues of the surrounding area. Particularly with the orbit, which has intrinsically delicate anatomy and perhaps potentially more devastating complications, this reduction in surgical insult and the ensuing inflammatory response may prove to be a significant benefit for the patient undergoing orbital floor reconstruction.

\section{Technique}

The patient is induced under general anesthesia with a nasotracheal or orotracheal intubation. Prophylactic antibiotics are administered intravenously and local anesthetic with epinephrine is injected into the site of the planned incision. The anterolateral sinus wall is exposed by an intraoral maxillary vestibular incision. A $1 \times 1 \mathrm{~cm}$ bony window is created on the anterolateral sinus wall. A $30^{\circ}$ endoscope is introduced into the sinus, and the defect and prolapsed orbital contents are visualized. The sinus roof is de-mucosalized, and any loose fragments of bone identified are removed. The orbital contents are reduced superiorly into the orbit using the blunt end of a periosteal elevator. At this point in the procedure, the next steps differ between the trans-antral only approach and the combination approach.

For the trans-antral only approach, the bony window must be large enough to allow for insertion of the implant. Common implants include a porous polyethylene implant trimmed about $4 \mathrm{~mm}$ larger than the defect or the use of a silastic sheet, which has the benefit of flexibility, which allows it to be inserted folded, and then unfolded once in the appropriate position (Figure 1A-1C).

For the combination approach, an inferior eyelid incision (subconjunctival vs. subciliary vs. subtarsal) is made. The orbital floor defect is identified and reconstructed using an appropriate implant (resorbable or non-resorbable plate or mesh). The endoscope is used trans-antrally to verify the correct position of the implant. The inferior eyelid incision is closed in layers.

For the antrostomy defect, if the shell of bone from the bony window was preserved, it may be secured back in position to the anterolateral maxillary sinus wall using a microplate and screws, or it may be left open. The maxillary vestibular incision is closed using resorbable sutures. Postoperatively, the patient is placed on sinus precautions, including postoperative antibiotics.

\section{Outcomes}

The trans-antral only approach spares the inferior eyelid incision and dissection through the periorbital tissue. This sparing of the inferior eyelid incision carries with it the significant benefit of preventing ectropion or entropion. The incidence of postoperative ectropion or entropion with conventional approaches may be as high as $4-5 \%(16,17)$. The mechanism by which ectropion or entropion may develop is either by the positioning of the tissues during closure and/or the effect of scar contracture after healing of the periorbital incision. By avoiding the inferior eyelid incision altogether, this complication is virtually reduced to $0 \%$ with the trans-antral only approach.

Both in the context of the trans-antral only approach and in combination with the open approach, the endoscopic approach to orbital floor reconstruction greatly improves visualization. As compared with conventional approaches, where visualization is down a long dark tunnel through the relatively small incision constantly obscured by the orbital contents, which must be continuously repositioned and retracted, the endoscopic approach allows very thorough visualization with relative ease. Among the aspects wellvisualized are the size and extent of the orbital floor defect, the size and position of any bone fragments which can then be removed with relative ease, and the positions of the posterior, medial, and lateral stops which must be ascertained for ideal modification and design of the orbital implant.

The endoscopic approach also affords the benefit of direct visual confirmation of several surgical objectives of the procedure. First is the ability to ensure that all orbital contents are above the orbital implant. Retropulsion of the globe while directly visualizing the reconstructed defect allows the surgeon to ensure that orbital fat and extraocular muscles are not impinged by the orbital implant. Next is the complete direct view of the seating of the orbital implant onto the posterior, medial and lateral stops. The direct visual confirmation of these elements provides an alternative 

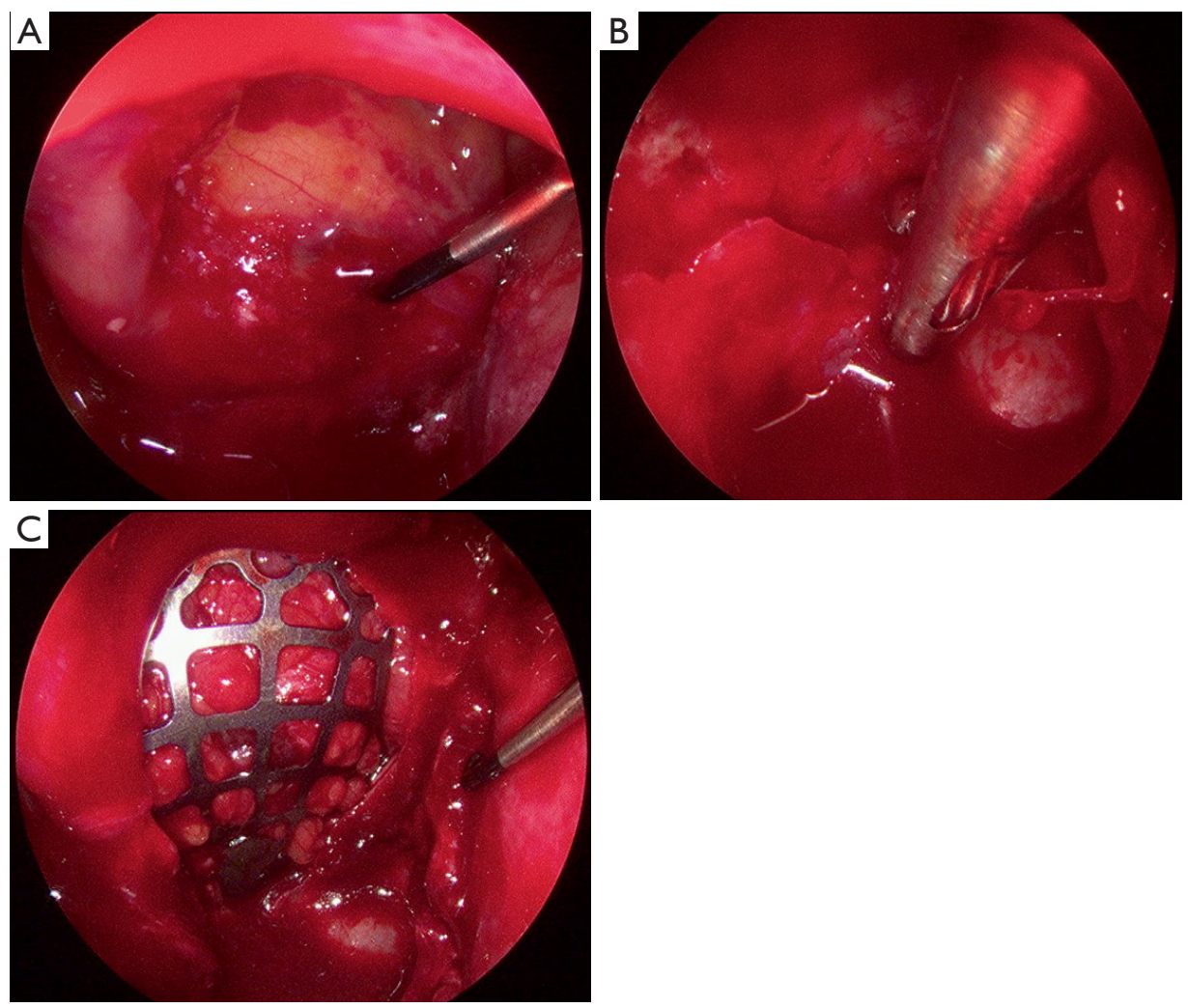

Figure 1 Reconstruction of an orbital fracture via a combination of trans-antral and subtarsal approaches. (A) Illustration of the orbital floor fracture with herniation of orbital contents into the maxillary sinus. (B) Removal of bony fragments with endoscopic instrumentation. (C) Confirmation of position of the orbital implant with an endoscope renders intraoperative CT unnecessary.

to the intraoperative CT scan which may not be available for various reasons, and which does carry an additional dose of radiation to the patient.

While outcome data for trans-antral approach is still relatively limited, multiple series have reported reasonably high rates of success. Farwell et al. analyzed 53 orbital floor repairs and their outcomes. Forty-five $(85 \%)$ were completed endoscopically and $8(15 \%)$ necessitated conversion to an open approach. Postoperatively, 36 of 45 patients $(80 \%)$ had no orbital complaints. Seven patients had postoperative diplopia that was improved from preoperative exam, 3 had enophthalmos, 2 had sinusitis requiring surgery, and 8 had persistence of their preoperative infraorbital hypesthesia (18). Ducic and Verret analyzed outcomes for 63 cases of endoscopic trans-antral repair of orbital floor fractures. No cases required conversion to an open approach. Two patients required a repeat repair. There were no cases of blindness, permanent new diplopia, ectropion, entropion, or new infraorbital anesthesia and no patients experienced any eyelid deformity after surgery (19). Polligkeit et al. analyzed 13 cases of endoscopy-assisted reconstruction of isolated orbital floor fractures via a combined subciliary and trans-antral approach. Their outcomes included 6 patients with residual postoperative diplopia, which did not affect the central $40^{\circ}$ of the visual field. Two patients had preoperative enophthalmos of $2 \mathrm{~mm}$ which had resolved postoperatively (20).

\section{Subcondylar fractures \& approach to ramus-condyle unit}

The use of the endoscopic technique in the repair of 
subcondylar fractures has the major benefits of reduced risk to the facial nerve as well as improved visualization at an anatomical site which is difficult to visualize with the naked eye. This improved visualization facilities both fracture assessment as well as assessment of fracture reduction and hardware adaptation at the site of fracture. With the endoscopic approach, the RCU can be accessed either through an intraoral incision or a trans-cervical incision with or without a trocar. When trans-cervical incisions are used, they can be made much smaller compared to traditional approaches, which avails the benefit of reduced risk of injury to the marginal mandibular branch of the facial nerve, as well as improved cosmesis. In a retrospective study of 40 patients who underwent endoscopic assisted transoral repair of fractures of the condylar process, Blumer et. al reported only one case of temporary facial palsy $(2.5 \%)$ which resolved after 3 months (21). This is in comparison to the overall rate of $5.8 \%$ treated with traditional approaches to open reduction and internal fixation (ORIF), $2.2 \%$ of which did not recover after 6 months (6 of 265 patients) (22).

\section{Technique}

The patient is induced under general anesthesia with a nasotracheal intubation. Prophylactic antibiotics are administered intravenously and local anesthetic with epinephrine is injected into the site of the planned incision. The patient is not placed into maxillomandibular fixation in order to allow for manipulation of the proximal and distal segments, facilitating reduction. For the transoral approach, a lateral vestibular incision is made similar to that of a sagittal split osteotomy (SSO). Subperiosteal dissection of the mandibular ramus is performed, creating a subperiosteal optical cavity. A $30^{\circ}$ angled endoscope is inserted to inspect and reduce the fracture. Osteosynthesis is performed using a trocar or an angulated screwdriver to place screws for rigid fixation (Figure $2 A, 2 B$ ). For the transcervical approach, the ascending ramus of the mandible is exposed after routine submandibular incision and dissection, and a subperiosteal optical cavity is developed on the lateral aspect of the ascending ramus. The endoscope is inserted and advanced until the fracture gap is visible. Osteosynthesis is achieved in the same manner as mentioned above (23-25) (Figure 2C,2D).

\section{Angle and body fractures of mandible}

One of the most significant challenges of treating mandibular body and angle fractures is limited access and visualization to this anatomic site. The use of the endoscope in the repair of mandibular body and angle fractures can greatly enhance visualization throughout the entire procedure. With the use of the endoscope, the surgeon and team can clearly visualize the fracture site and reduction as well as examination of any bony segments. Once the fractures are reduced, the endoscope can be used to confirm ideal hardware contouring, adaptation, and positioning. This can be particularly helpful in visualization of the inferior and posterior borders which are typically some of the hardest anatomic structures to visualize. Upon completion of the fixation, the endoscope can be used to visualize and examine the occlusion, ensuring complete interdigitating of occlusion. And finally, as with all endoscopic procedures, the technique avails easy and efficient photo and video documentation of aspects of the procedure and anatomy that are otherwise only viewable to the primary surgeon.

\section{Technique}

The technique for endoscopic-assisted ORIF of mandibular angle and body fractures is similar to that previously described for subcondylar fractures. An incision is made, similar to the standard intraoral incision for ORIF of the mandibular angle, but which can be made smaller because of the use of the endoscope for visualization. Subperiosteal dissection is performed, creating an optical cavity. A $30^{\circ}$ endoscope is introduced, the fractures are visualized and reduced, and fixation plates are positioned and fixated using a trocar technique (23) (Figure $3 A, 3 B$ ).

\section{Frontal sinus fractures}

Treatment of frontal sinus fractures can be broadly categorized into three specific treatments: repair of anterior frontal bone table fractures, obliteration, and cranialization. Treatment selection is largely contingent upon: patency of the nasofrontal duct, degree of deformity, and presence of posterior table fractures and cerebral/dural injury. Open surgical approaches to repair these fractures including coronal, open-sky, or gull-wing approaches (the latter two render poor cosmetic outcomes). Complex fracture patterns require advanced reduction and fixation to achieve stable and esthetic outcomes should be treated via open approaches via coronal or through existing lacerations. In instances of a depressed anterior table fracture, no significant comminution and patency of the nasofrontal 

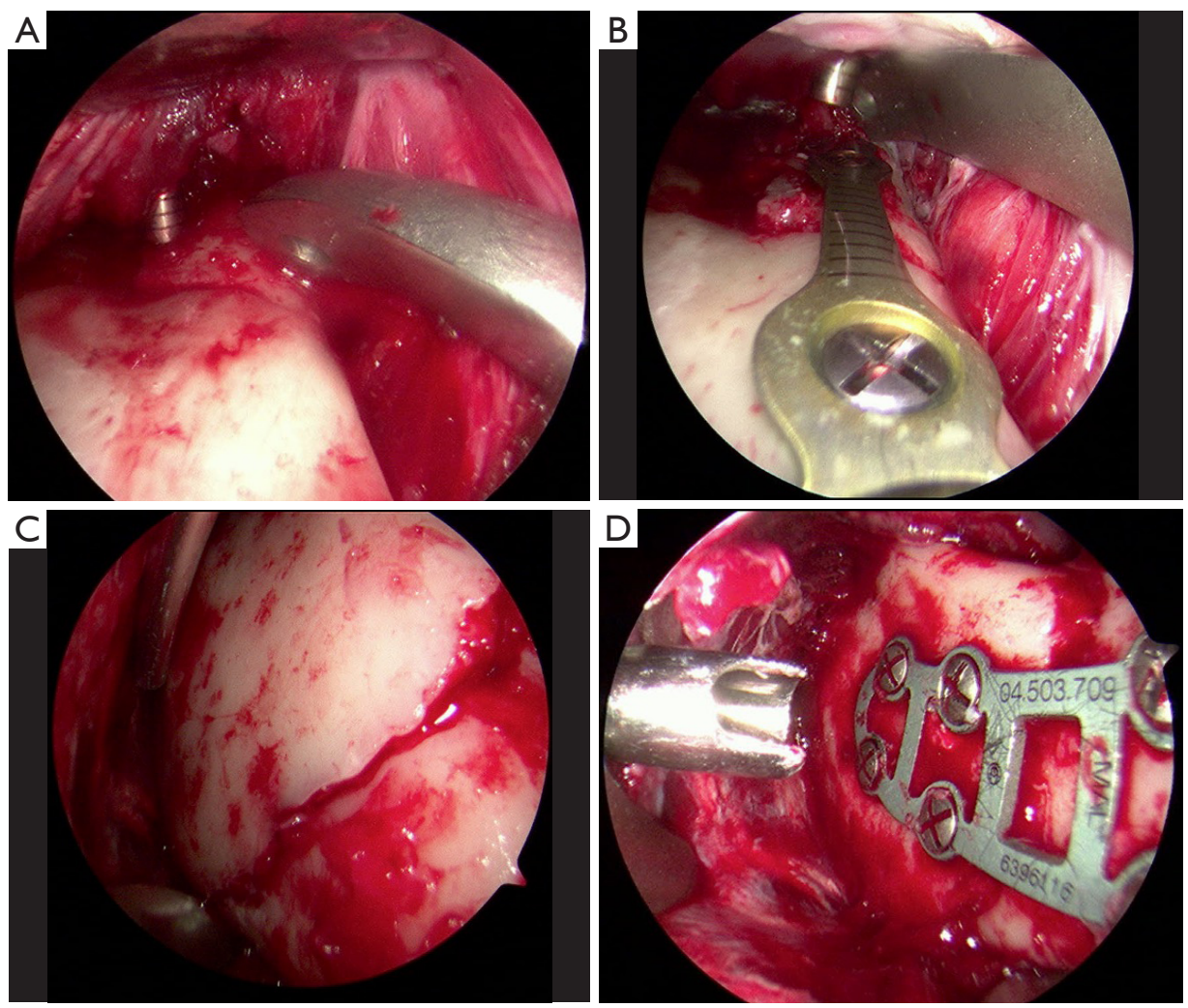

Figure 2 Endoscopic-assisted ORIF of left subcondylar fracture and right mandibular angle fracture. (A) Visualization and reduction of left subcondylar fracture via a trans-cervical approach. (B) Placement and confirmation of left subcondylar hardware. (C) Visualization and reduction of right mandibular angle fracture. (D) The use of an endoscope facilitates placement of right mandibular angle hardware. ORIF, open reduction and internal fixation.

duct, a minimally invasive endoscopic approach, reduction, and fixation of the fracture (with sinus preservation) can be successful and predictable $(26,27)$.

\section{Technique}

A $30^{\circ}$ endoscope is entered through either existing lacerations present in the soft tissue drape of the forehead, or through stab incisions made across the hairline (in similar fashion as conducted in an endoscopic brow lift procedures). Benefits of the endoscopic minimally invasive approach include avoidance of large incisions leading to improved patient recovery, elimination of the risk of strip alopecia from rainy clips, reduced paresthesia, limited to no scar formation, and avoidance of nerve injury. The endoscope also can be used to evaluate the integrity of the nasofrontal duct in detail via sinuscopy once entered into the frontal sinus. The diagnostic and treatment advantage endoscopy provides in ensuring patency of the nasofrontal duct has been shown to be a significant factor in frontal sinus preservation (28). Under direct visualization through the endoscope, the fractured anterior table segments are reduced with instruments (typically a periosteal elevator or nerve hook) placed through distant stab incisions within the hairline. Fixation can be accomplished, by making additional stab incision(s) within the eyebrows (or using existing lacerations), taking care to prevent incising hair follicles, to achieve straight-line access to place a drill sleeve to drill holes and place screws to secure miniplate fixation to reconstruct the anterior table. When conducting obliteration operations under open technique, the endoscope provides assistances in evaluating all internal aspects of the frontal sinus to ensure all sinus mucosa is removed, and also to evaluate the posterior wall of the sinus after peripheral ostectomy. Similar to the endoscope's efficacy in evaluating patency of the nasofrontal duct, during obliteration cases, the scope can be navigated to inspect and ensure that the duct is completely sealed via autogenous or alloplastic means (such as fibrin glue) (23,26-28). 

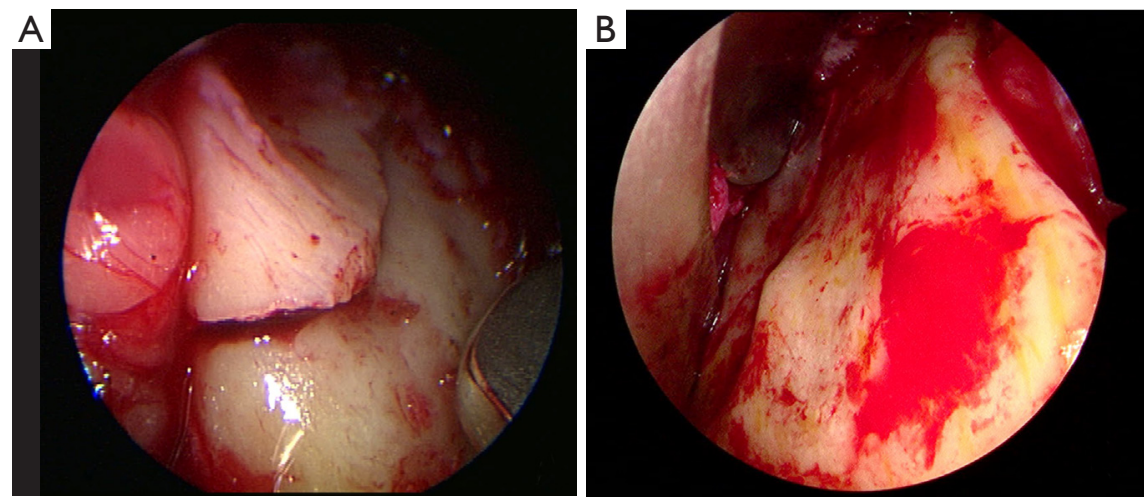

Figure 3 Endoscopic view of a subcondylar fracture. (A) Illustrating the fracture via a submandibular approach, where the posterior border of the mandible is well visualized. (B) Demonstrating the fracture via an intraoral approach, where visualization of the posterior border of the mandible is limited.

\section{Review of applications in other oral and maxillofacial surgery procedures}

\section{Removal of foreign bodies/sinuscopy}

The endoscope can provide a very useful diagnostic and operative aid in locating and removing foreign bodies that are dislodged into the maxillary sinus. The sinus, given it's hallow bony architecture, is a natural optical cavity. In the event that a tooth or other foreign body (such as a broken instrument) is dislodged into the antrum, the endoscope can be entered through the defect to locate and assist in removal of the tooth/foreign body. The endoscope is also used commonly to assist in conducting a Caldwell Luc approach and/or sinuscopy when the anterior wall of the antrum is fractured due to trauma to remove intruded teeth or a foreign body/debris. The endoscope may also be used to visualize and inspect the infundibulum and osteon in the medial antral wall. Typically a $30^{\circ}, 4.0 \mathrm{~mm}$ endoscope is used to conduct sinuscopy. This allows for an enhanced field of view and the scope is rigid enough to be safely navigated amongst fractured osseous segments to prevent breakage. The ability to easy and efficiently perform sinuscopy can be of great diagnostic and operative benefit to the oral and maxillofacial surgeon in both the outpatient clinic and main operating room settings.

\section{Orthognatbic surgical applications}

The endoscope is useful in orthognathic surgery to enhance visualization during crucial steps to help reduce the chance of complications. In the SSO, the endoscope can be used to confirm appropriate positioning and depth of the medial osteotomy as well as the inferior border osteotomy, both of which can contribute to unfavorable fractures if insufficient or improperly positioned. A challenge of the vertical ramus osteotomy is the appropriate anteroposterior positioning of the osteotomy. An osteotomy too far anteriorly can result in transection of the inferior alveolar nerve, whereas too far posteriorly can result in a subcondylar osteotomy. Using the endoscope to confirm positioning before making the osteotomy can help prevent these complications. In the LeFort I osteotomy, the endoscope can be a valuable teaching tool to allow observers to see what typically is only available for the surgeon's eyes, in addition to providing enhanced visualization of anatomical structures that even the primary surgeon is not often able to clearly visualize. Some of these regions and structures in particular include the posterior maxilla, the pterygomaxillary junction, pterygoid plates, and nasal septum.

\section{Sialoendoscopy}

Sialoendoscopy enables the diagnosis and treatment of obstructive salivary gland disease while sparing the patient the potential complications of open techniques including scarring, nerve damage, hemorrhage, xerostomia, and gustatory sweating.

Sialoendoscopy starts with cannulation of the duct using dilating probes. Once the duct is adequately dilated, the endoscope is introduced into the duct, using forceps to maintain counter tension. Isotonic saline irrigation facilitates navigation through the ductal system as well 
as flushing out any debris encountered. If visualized, an obstruction may be retrieved or instrumented using a basket, clasp, Fogarty catheter, or holmium laser (HL) for laser lithotripsy. A 2-mm polyethylene stent is placed into the duct and sutured into place for 4 weeks postoperatively to help prevent ductal stenosis.

Most complications are considered minor, and have been reported to have an incidence less than 10\% (29). Complications include swelling of the gland, extravasation of irritant into the floor of the mouth and surrounding tissues, iatrogenic perforation of the duct, transient paresthesia to the lingual nerve, iatrogenic ranula, infection, trauma to the papilla, and stricture of the duct.

\section{Open arthrotomy and related TMJ procedures}

Open arthrotomy and total alloplastic joint reconstructive procedures are greatly enhanced through the application of endoscope assistance. Given the enhanced visualization of the surgical site with navigation of the endoscope, smaller incisions can be conducted to accomplish these procedures. In addition, once the joint space is encountered, the endoscope is able to be traversed within the joint space to survey all anatomical structures and surfaces, and to assist with visualizing surgical maneuvers. This is especially useful in the medial aspect of the joint, and under-surface of the glenoid fossa (GF)-articular eminence complexwhich can be difficult to visualize and inspect with the naked eye. Another useful endoscopic correlate during open arthrotomy-discectomy and gap-arthroplasty procedures involves maneuvering the endoscope throughout the joint space to ensure all articular disc and synovial tissues have been resected. The intervening joint-space gap filling material (such as temporalis muscle/fascia, fat, or temporary alloplastic material such as a silastic implant) can be clearly visualized with the endoscope. This ensures ideal positioning, and fixation of the material to fill the joint space as intended by the operating surgeon. Efficient and sterile photo and video documentation can be easily obtained.

\section{TMF total joint replacement (THR)—review of principles and technique}

There are multiple benefits to the implementation of maxillofacial endoscopy assistance during the TMJ TJR surgical procedure. Through a combination of direct and endoscopic visualization, the operating surgeon and team can visualize the adaptation of cutting guides onto the RCU from all aspects. The ostomy site subsequently can be visualized and inspected from multiple angles. This ensures that bone resection is conducted in coordination with pre-surgical planning specifications. This preserves bone stalk and empirically ensures ideal preparation of the facial skeleton to receive subsequent hardware. After the osteotomy of the condyle is conducted, the endoscope can assist in visualizing and ensuring that blood vessels and surrounding soft tissues are retracted and protected prior to delivering the resected condyle. This prevents inadvertent damage and bleeding to these surrounding structures. Assessment of the joint space is paramount to ensure that osseous architecture is adequately prepared, and no intervening soft tissue (synovium) is present. The scope is especially useful in inspecting the medial aspect of the TMJ and undersurface of the fossa-eminence complex in great detail (Figure 4A-4D).

Once the alloplastic or autogenous [costochondral graft (CCG)] joint components are seated, the endoscope in conjunction with direct observation can be navigated from all aspects around the fossa and condylar prostheses (or CCG) to ensure ideal positioning and adaptation. The articulation can also be visualized, photographed, and video-recorded in detail during static and dynamic function prior to wound closure.

\section{Coronoidectomy — review of principles and technique}

Coronoidectomy is a procedure that is commonly performed as an isolated operation, or in conjunction with TMJ TJR. Common indications for this procedure include patients presenting with mandibular hypomobility due to coronoid impingement, severe traumatic injuries, and refractory TMJ ankyloses due to temporalis muscle contracture (30). Utilization of the endoscope allows surgeons to visualize the coronoid process via trans-oral approach to ensure osteotomy is localized in an ideal position to ensure adequate bone stock is removed $(31,32)$. Under direct and endoscopic assisted visualization, the sigmoid notch is dissected in the subperiosteal plane and visualized, and a nerve-hook is placed into the notch. Subsequently, a through-and-through bur-hole is drilled through the distal aspect of the coronoid process - superior to the planned osteotomy site. A 24-guage wire is then passed through the bur-hole to act as a tether cord to prevent superior migration of the bone segment if inadvertent release of the segment occurs during definitive dissection of the superior temporalis muscle tendinous attachment. The endoscope assists in ensuring ideal osteotomy placement, protection 

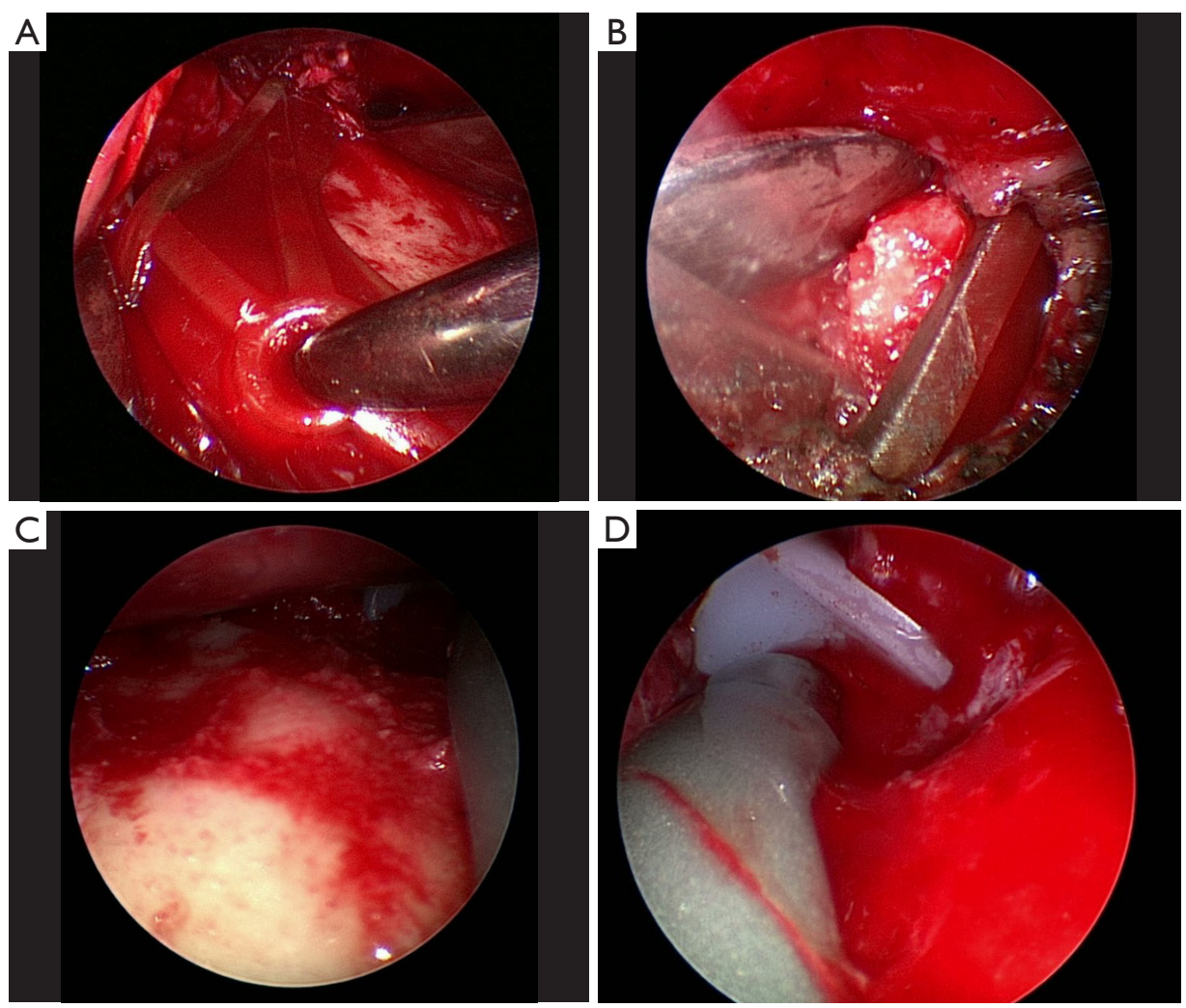

Figure 4 Endoscopic assisted TMJ TJR. (A) Endoscopic assisted visualization of circumferential seating of the ramal cutting guide to ensure ideal adaptation in conjunction with pre-surgical planning. (B) Superior view of condylar resection margin. Note ideal adaptation of the cutting guide is well visualized. (C) Endoscopic view of the osteotomized ramus, in preparation to receive TJR. Adequate anatomical space has been created. The scope is placed within the subperiosteal pocket to establish an optical cavity. (D) Evaluation of articulation of the condylar and fossa components of the TMJ TJR. This view is unable to be evaluated with direct visualization of the surgeon, and only made possible with use of the endoscope. This view, in conjunction with the pre-auricular direct view ensure ideal seating and articulation of the condylar prosthesis within the fossa prosthesis. TMJ, temporomandibular joint; TJR, total joint replacement.

and retraction of surrounding soft tissues, and complete dissection of temporalis muscle attachment, facilitating precise removal of the coronoid process, with limited trauma to surrounding tissues.

\section{Eminectomy—review of principles and technique}

Eminectomy is the surgical reduction of the articular eminence of the TMJ which is most commonly conducted for treatment of chronic condylar dislocation or closed locking of the mandible (33). Many of the advantages afforded by maxillofacial endoscopy during TJR GF preparation and visualization also pertain to performing eminectomy. The endoscope can be navigated to inspect all aspects of the fossa-eminence complex. This is especially useful in inspecting the medial aspect of the joint space, which can be difficult to visualize with the eye, to prevent bleeding from inadvertently vascular injury. During otectomy, direct and endoscopic visualization of the eminence ensure that adequate bone removal is conducted. One can determine the amount of bone removal relative to the greatest concavity of the GF using the endoscope. This also provides a critical safety measure to reduce the likelihood of excessive bone removal and perforation of the cranial base into the middle cranial fossa. The endoscope is also invaluable in ensuring adequate removal of the articular tubercle of the articular eminence-which is medially located deep within the GF-eminence complex. This minimizes the likelihood of refectory condylar dislocation after the procedure is completed. Finally, the 

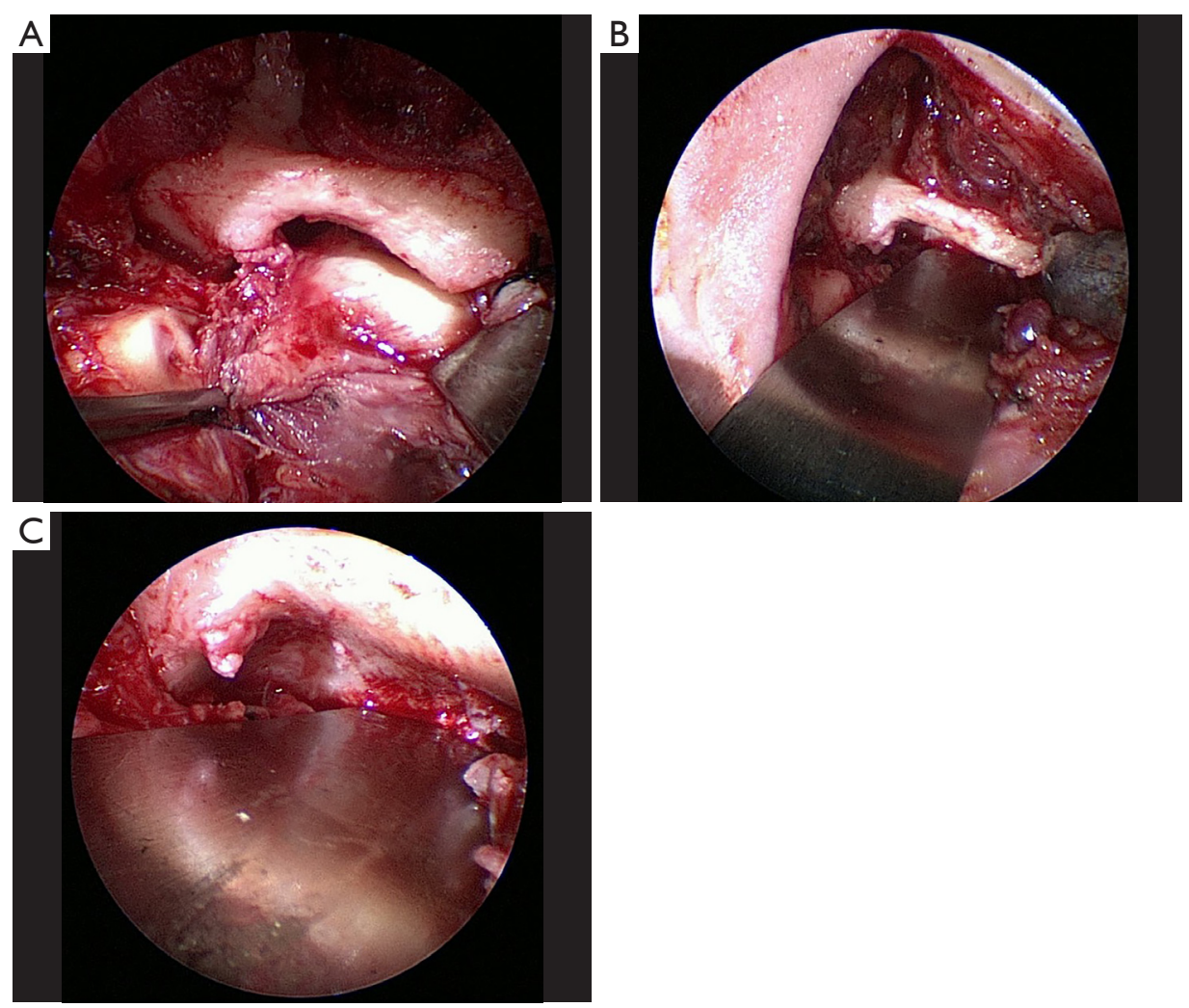

Figure 5 Endoscopic assisted eminectomy. (A) Pre-operative eminectomy in condylar dislocation case-prior to ostectomy removal of the articular eminence-gross anatomical view. (B) Post-operative eminectomy procedure after ostectomy conducted with removal of articular eminence with direct and endoscopic visualization. (C) Intra-articular endoscopic examination after surgical removal of the articular eminence, to ensure all contours are flattened to prevent recurrent condylar dislocation.

patient's mandible is functioned (and video recorded with the endoscope) at the conclusion of the procedure to ensure resolution of condylar dislocation or closed locking of mandible does not occur (Figure 5A-5C).

\section{TMJ arthroscopy}

Arthroscopy of the TMJ allows for minimally invasive diagnosis and surgical management of intra-articular disorders. The efficacy and established positive outcomes of this surgical modality in treating a wide-range of intraarticular maladies have been vetted and reported globally (34-40). Although minor variations in armamentarium exist between providers, the arthroscope most commonly employed for this procedure is small-ranging from $1.2-2.4 \mathrm{~mm}$ in size; rigid; and, is commonly angled with a $30^{\circ}$ field of view. Levels of operative sophistication and complexity exist in TMJ arthroscopy which denotes the type of intra-articular procedures that can be conducted. These levels of TMJ arthroscopic surgery can be classified utilizing the McCain Classification (15).

\section{Technique}

* Level I: single-puncture arthroscopic portal into the posterior recess of the superior joint space of the TMJ, in conjunction with an outflow needle. This facilitates arthroscopic arthrocentesis, which allows for lysis and lavage along with diagnostic visualization and examination of intra-articular anatomical structures utilizing the arthroscope. The seven points of interest can be visualized when conducting a diagnostic sweep throughout the joint [(I) medial synovial drape, (II) pterygoid shadow, (III) retro-discal synovium, (IV) GF and posterior slope of the articular-eminence complex, (V) articular disc, (VI) intermediate zone, 


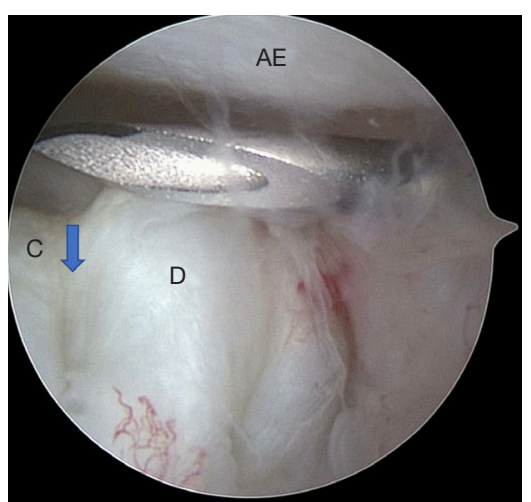

Figure 6 Level I arthroscopy of right TMJ. A single puncture into the posterior pouch of the joint with outflow needle for arthroscopic arthrocentesis is visualized. C: condylar head, visible through articular disc perforation; D: articular disc with perforation-arrow denotes perforation margin; AE: posterior slope of the articular eminence. TMJ, temporomandibular joint.

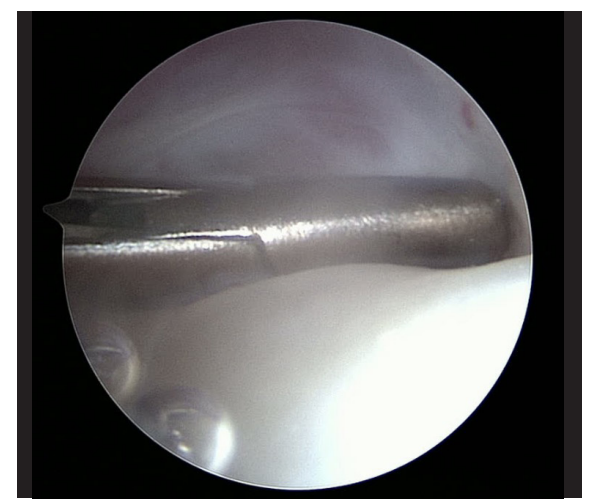

Figure 7 Level II arthroscopic TMJ surgery demonstrating obtaining a synovial biopsy utilizing the Ergo forceps. This instrument is implemented after a stab incision is made in the synovial lining to obtain a representative synovial biopsy. TMJ, temporomandibular joint.

and (VII) anterior recess] (15) (Figure 6).

* Level II: a second puncture is conducted, consisting of an operative cannula (OC). The importance of portal placement is paramount to the success of intraarticular arthroscopic surgery. The OC is introduced into the joint within the anterior recess implementing triangulation vector measuring technique (Figure 7). Level II TMJ arthroscopic surgery allows surgeons to accomplish the following procedures:

- Lysis of adhesions under direct arthroscopic visualization;

- Synovial biopsy to assist in enhanced diagnosis of underlying pathology;

- Debridement of arthritis joints to achieve orthopedic arthroscopic surgical principles: smoothening intraarticular surfaces, and increasing joint space;

- Targeted deposition of medications for disease modification and influencing intra-articular chemistry;

- Retro-discal tissue (RDT) contracture to prevent condylar dislocation in cases of hypermobility, and reduction of redundant synovium (15).

* Level III: arthroscopic articular disc reduction and fixation (discopexy). This includes conducting a myotomy of the superior belly of the lateral pterygoid (SBLP) muscle attachment onto an anteriorly displaced articular disc, disc reduction, contracture of RDTs, and fixation of the articular disc.

* Advanced arthroscopic debridement of fibrous ankyloses and articular disorder Wilkes $\mathrm{V}$ cases (15) (Figure 8A-8G).

\section{Outcomes}

The goal of arthroscopic surgical procedures is to obtain the consistent or improved outcomes relative to open traditional procedures, in minimally invasive fashion. This achieves reduced patient morbidity and optimizes healing, expediting return to function. Traditional open surgical techniques for articular disc fixation (discopexy), treatment of recurrent condylar dislocation, and many open arthrotomy procedures are being routinely supplanted by minimally invasive established arthroscopic surgical interventions with excellent outcomes to date (34,39,41-43). Procedures to achieve arthroscopic orthopedic principles of increasing joint space, smoothening articular surfaces for improved range of motion, smooth articulation, and altering intra-articular joint chemistry from a catabolic to anabolic status are routinely performed with consistent outcomes (35-40). Revitalization of the intra-articular fibrocartilage lining of the joint in cases with chondromalacia by implementing arthroscopic microfracture techniques have also shown promise and efficacy $(44,45)$.

Further prospective studies are needed comparing advanced arthroscopic surgery (levels II and III) to single puncture (level I) arthroscopic interventions. Martin Granizo and colleagues performed advanced arthroscopy on 659 patients. A total of $5.9 \%$ of the patients treated 

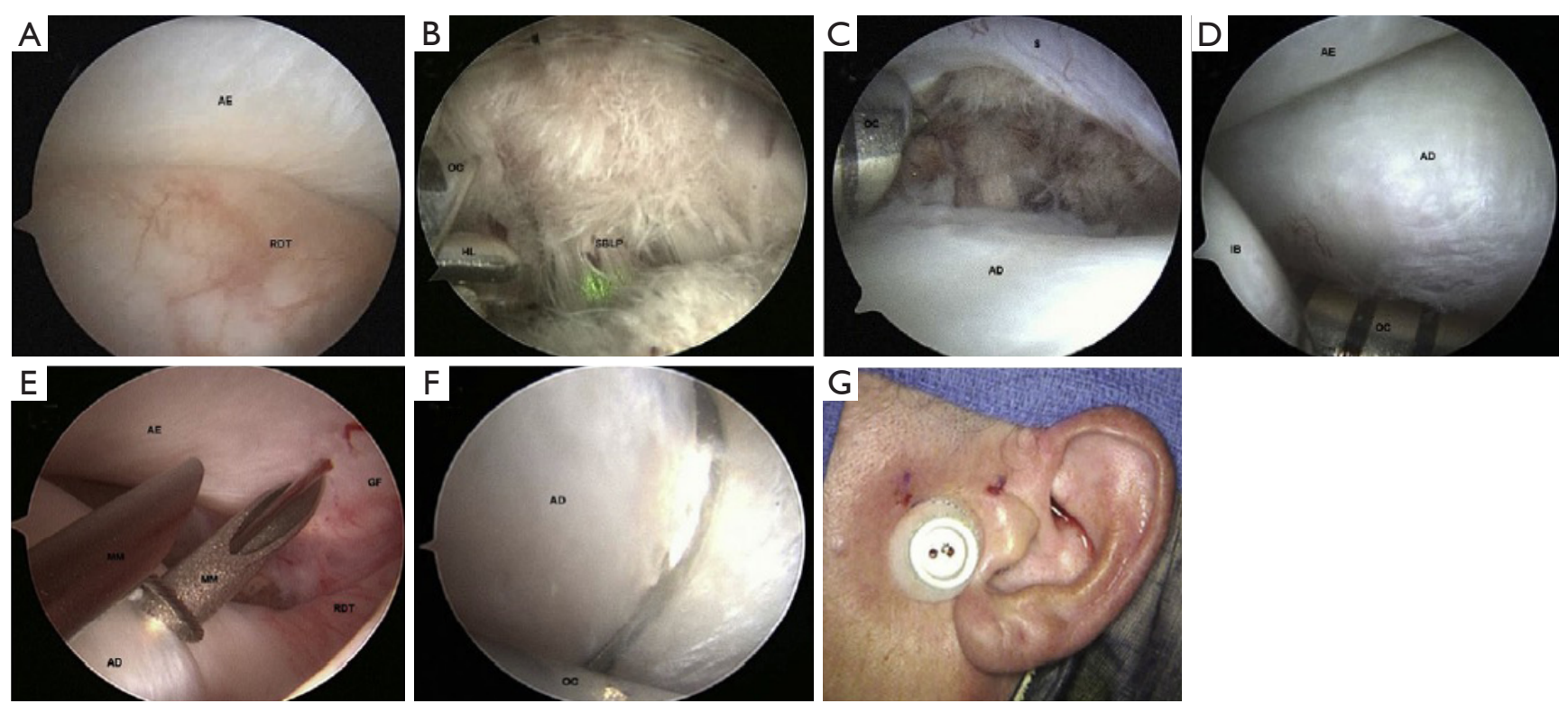

Figure 8 Level III arthroscopic discopexy of the left TMJ; open mouth position demonstrates RDT under the AE, indicating an anteriorly dislocated disc. Notice the hyperemia of the RDT (A). The OC is inserted into the most anterolateral portion of the anterior recess, and $\mathrm{HL}$ is used to cut the attachment of the SBLP to the AD. (B) Zoomed out photograph demonstrates separation of the S from the AD, which gave access to the lateral pterygoid muscle, which has been incised in this picture (C). The OC is used to reduce the AD so it lies under the AE. IB can form during arthroscopy (D). The RDT has been reduced posteriorly under the GF. The disc is held in reduction with the OC, while a MM is inserted through the skin to lightly contact the condyle and then directed superiorly through the posterolateral portion of the disc; then, a 28-gauge wire is passed through it. Another MM is inserted directly into the superior joint space, and a lasso is used to capture the wire. The MMs are withdrawn to leave the two ends of the wire on the external surface of preauricular region (E). The OC is moved above the wire to hold the disc in reduction (F), while the wire is externally tightened through a button (G). The wire and button are typically left in place for 2 to 3 weeks and then removed in office. Reprinted from (15). TMJ, temporomandibular joint; RDT, retrodiscal tissue; OC, operative cannula; HL, holmium laser; SBLP, superior belly of the lateral pterygoid; AD, articular disc; S, synovium; AE, articular eminence; IB, irrigation bubbles; GF, glenoid fossa; MM, meniscus mender.

required additional arthroscopic intervention (46). Breik and colleagues conducted a level I arthroscopy study of 167 patients which demonstrated a reoperation rate $22 \%$ (47). The advanced surgical interventions and affection on intra-articular structures achieved by advanced operative arthroscopy (levels II and III) are suspected to contribute to this discrepancy in re-operation rates. A definitive randomized clinical trial comparing single puncture (level I) to advanced arthroscopic surgery (II-III) is indicated for further study and evaluation.

\section{Summary}

The incorporation endoscopy into oral and maxillofacial surgical procedures has enhanced our diagnostic and surgical capabilities and skillset in the care of patients. Oral and maxillofacial surgeons are poised to continue to implement and discover novel applications for endoscopically assisted and facilitated maxillofacial surgical procedures. Decreased complication rates, comparable success rates, diverse functionality, and efficiency render the endoscope an indispensable instrument in the modern surgeon's armamentarium.

\section{Acknowledgments}

Funding: None.

\section{Footnote}

Provenance and Peer Review: This article was commissioned by the Guest Editor (Sung-Kiang Chuang) for the series "Clinical Outcomes and Innovations in Oral and Maxillofacial Surgery" published in Frontiers of Oral and 
Maxillofacial Medicine. The article has undergone external peer review.

Conflicts of Interest: All authors have completed the ICMJE uniform disclosure form (available at https://fomm. amegroups.com/article/view/10.21037/fomm-21-12/coif). The series "Clinical Outcomes and Innovations in Oral and Maxillofacial Surgery" was commissioned by the editorial office without any funding or sponsorship. The authors have no other conflicts of interest to declare.

Ethical Statement: The authors are accountable for all aspects of the work in ensuring that questions related to the accuracy or integrity of any part of the work are appropriately investigated and resolved.

Open Access Statement: This is an Open Access article distributed in accordance with the Creative Commons Attribution-NonCommercial-NoDerivs 4.0 International License (CC BY-NC-ND 4.0), which permits the noncommercial replication and distribution of the article with the strict proviso that no changes or edits are made and the original work is properly cited (including links to both the formal publication through the relevant DOI and the license). See: https://creativecommons.org/licenses/by-nc-nd/4.0/.

\section{References}

1. De Groen PC. History of the endoscope [scanning our past]. Proceedings of the IEEE 2017;105:1987-95.

2. Verger-Kuhnke AB, Reuter MA, Beccaria ML. Biography of Phillip Bozzini (1773-1809) an idealist of the endoscopy. Actas Urol Esp 2007;31:437-44.

3. Paraskeva PA, Nduka CC, Darzi A. The evolution of laparoscopic surgery. Minimally Invasive Therapy 1994;3:69-75.

4. Bircher E. Die arthroendoskopie. Zentralbl Chir 1921;48:1460-1.

5. Nitzan DW, Dolwick MF, Heft MW. Arthroscopic lavage and lysis of the temporomandibular joint: a change in perspective. J Oral Maxillofac Surg 1990;48:798-801; discussion 802.

6. Papadaki ME, McCain JP, Kim K, et al. Interventional sialoendoscopy: early clinical results. J Oral Maxillofac Surg 2008;66:954-62.

7. Kellman RM. Endoscopically assisted repair of subcondylar fractures of the mandible: an evolving technique. Arch Facial Plast Surg 2003;5:244-50.

8. Troulis MJ, Kaban LB. Endoscopic approach to the ramus/ condyle unit: Clinical applications. J Oral Maxillofac Surg 2001;59:503-9.

9. Alley JR Jr, Stucky CC, Moncure M. Teaching surgical residents dome-down laparoscopic cholecystectomy in an academic medical center. JSLS 2008;12:368-71.

10. Fritsch MH. A new sialendoscopy teaching model of the duct and gland. J Oral Maxillofac Surg 2008;66:2409-11.

11. Li H, Zheng J, Cai JY, et al. Laparoscopic VS open hepatectomy for hepatolithiasis: An updated systematic review and meta-analysis. World J Gastroenterol 2017;23:7791-806.

12. Williams WB, Abukawa $\mathrm{H}$, Shuster $\mathrm{V}$, et al. A comparison of postperative edema after introral vs. endoscopic mandibular ramus osteotomy. J Oral Maxillofac Surg 2003;8:61a-2.

13. Kim K, McCain JP. Use of the endoscope in bisagittal split osteotomy. J Oral Maxillofac Surg 2008;66:1773-5.

14. Westphal D, Kreidler JF. Sinuscopy for the diagnosis of blow-out fractures. J Maxillofac Surg 1977;5:180-3.

15. Hakim MA, McCain JP, Ahn DY, et al. Minimally Invasive Endoscopic Oral and Maxillofacial Surgery. Oral Maxillofac Surg Clin North Am 2019;31:561-7.

16. Yamashita $M$, Kishibe $M$, Shimada K. Incidence of lower eyelid complications after a transconjunctival approach: influence of repeated incisions. J Craniofac Surg 2014;25:1183-6.

17. Ridgway EB, Chen C, Lee BT. Acquired entropion associated with the transconjunctival incision for facial fracture management. J Craniofac Surg 2009;20:1412-5.

18. Farwell DG, Sires BS, Kriet JD, et al. Endoscopic repair of orbital blowout fractures: use or misuse of a new approach? Arch Facial Plast Surg 2007;9:427-33.

19. Ducic Y, Verret DJ. Endoscopic transantral repair of orbital floor fractures. Otolaryngol Head Neck Surg 2009;140:849-54.

20. Polligkeit J, Grimm M, Peters JP, et al. Assessment of indications and clinical outcome for the endoscopy-assisted combined subciliary/transantral approach in treatment of complex orbital floor fractures. J Craniomaxillofac Surg 2013;41:797-802.

21. Blumer M, Guggenbühl T, Wagner MEH, et al. Outcome of Surgically Treated Fractures of the Condylar Process by an Endoscopic Assisted Transoral Approach. J Oral Maxillofac Surg 2019;77:133.e1-9.

22. Al-Moraissi EA, Ellis E 3rd. Surgical treatment of adult mandibular condylar fractures provides better outcomes than closed treatment: a systematic review and metaanalysis. J Oral Maxillofac Surg 2015;73:482-93.

23. Pedroletti F, Johnson BS, McCain JP. Endoscopic techniques in oral and maxillofacial surgery. Oral 
Maxillofac Surg Clin North Am 2010;22:169-82.

24. Schön R, Schmelzeisen R. Endoscopic fracture treatment. Ann R Australas Coll Dent Surg 2002;16:40-5.

25. Chen CT, Lai JP, Tung TC, et al. Endoscopically assisted mandibular subcondylar fracture repair. Plast Reconstr Surg 1999;103:60-5.

26. Johnson NR, Roberts MJ. Frontal sinus fracture management: a systematic review and meta-analysis. Int J Oral Maxillofac Surg 2021;50:75-82.

27. Bell RB. Management of frontal sinus fractures. Oral Maxillofac Surg Clin North Am 2009;21:227-42.

28. Elkahwagi M, Eldegwi A. What Is the Role of the Endoscope in the Sinus Preservation Management of Frontal Sinus Fractures? J Oral Maxillofac Surg 2020;78:1811.e1-9.

29. Nahlieli O, Baruchin AM. Long-term experience with endoscopic diagnosis and treatment of salivary gland inflammatory diseases. Laryngoscope 2000;110:988-93.

30. Kaban LB, Perrott DH, Fisher K. A protocol for management of temporomandibular joint ankylosis. J Oral Maxillofac Surg 1990;48:1145-51; discussion 1152.

31. Robiony M, Casadei M, Costa F. Minimally invasive surgery for coronoid hyperplasia: endoscopically assisted intraoral coronoidectomy. J Craniofac Surg 2012;23:1838-40.

32. Mavili E, Akyürek M, Kayikçioğlu A. Endoscopically assisted removal of unilateral coronoid process hyperplasia. Ann Plast Surg 1999;42:211-6.

33. McCain JP, Hossameldin RH. Eminectomy. In: Haggerty CJ, Laughlin RM. editors. Atlas of operative oral and maxillofacial surgery. Hoboken: John Wiley \& Sons, Inc., 2015:chapter 34.

34. McCain JP, Hossameldin RH, Srouji S, et al. Arthroscopic discopexy is effective in managing temporomandibular joint internal derangement in patients with Wilkes stage II and III. J Oral Maxillofac Surg 2015;73:391-401.

35. Hossameldin RH, McCain JP. Outcomes of office-based temporomandibular joint arthroscopy: a 5-year retrospective study. Int J Oral Maxillofac Surg 2018;47:90-7.

36. Choi DD, Vandenberg K, Smith D, et al. Is Temporomandibular Joint Arthroscopy Effective in Managing Pediatric Temporomandibular Joint Disorders in the Short- and Long-Term? J Oral Maxillofac Surg 2020;78:44-51.

doi: $10.21037 /$ fomm-21-12

Cite this article as: Fanelli CA, Vera LC, Ahn DY, McCain JP. A review of endoscopic surgical applications in oral and maxillofacial surgery. Front Oral Maxillofac Med 2023;5:17.
37. Israel HA, Behrman DA, Friedman JM, et al. Rationale for early versus late intervention with arthroscopy for treatment of inflammatory/degenerative temporomandibular joint disorders. J Oral Maxillofac Surg 2010;68:2661-7.

38. Muñoz-Guerra MF, Rodríguez-Campo FJ, Escorial Hernández V, et al. Temporomandibular joint disc perforation: long-term results after operative arthroscopy. J Oral Maxillofac Surg 2013;71:667-76.

39. McCain JP, Sanders B, Koslin MG, et al. Temporomandibular joint arthroscopy: a 6-year multicenter retrospective study of 4,831 joints. J Oral Maxillofac Surg 1992;50:926-30.

40. Silva PA, Lopes MT, Freire FS. A prospective study of 138 arthroscopies of the temporomandibular joint. Braz J Otorhinolaryngol 2015;81:352-7.

41. Liu X, Zheng J, Cai X, et al. Techniques of Yang's arthroscopic discopexy for temporomandibular joint rotational anterior disc displacement. Int J Oral Maxillofac Surg 2019;48:769-78.

42. Ohnishi M. Arthroscopic surgery for hypermobility and recurrent mandibular dislocation. Oral Maxillofac Surg Clin North Am 1989;1:153.

43. Torres DE, McCain JP. Arthroscopic electrothermal capsulorrhaphy for the treatment of recurrent temporomandibular joint dislocation. Int J Oral Maxillofac Surg 2012;41:681-9.

44. Knutsen G, Drogset JO, Engebretsen L, et al. A randomized trial comparing autologous chondrocyte implantation with microfracture. Findings at five years. J Bone Joint Surg Am 2007;89:2105-12.

45. Naujoks C, Meyer U, Wiesmann HP, et al. Principles of cartilage tissue engineering in TMJ reconstruction. Head Face Med 2008;4:3.

46. Martin Granizo R, Correa Muñoz DC, Varela Reyes E. Rearthroscopy of the temporomandibular joint: A retrospective study of 600 arthroscopies. J Craniomaxillofac Surg 2018;46:1555-60.

47. Breik O, Devrukhkar V, Dimitroulis G. Temporomandibular joint (TMJ) arthroscopic lysis and lavage: Outcomes and rate of progression to open surgery. J Craniomaxillofac Surg 2016;44:1988-95. 